\title{
Empfehlungen der Netzhautkommission der ÖOG zur Durchführung einer IVOM (intravitreale operative Medikamentenapplikation) 2017
}

\author{
Gerhard Kieselbach für die Netzhautkommission der ÖOG 2017
}

Eingegangen: 5. Oktober 2018 / Angenommen: 16. Oktober 2018 / Online publiziert: 8. November 2018

(c) Der/die Autor(en) 2018

\begin{abstract}
Zusammenfassung Um eine intravitreale operative Medikamentenapplikation (IVOM) lege artis durchzuführen, werden mittels dieser Empfehlung der Netzhautkommission der Österreichischen Ophthalmologischen Gesellschaft (ÖOG) die wesentlichen, durch Studien belegte Punkte hervorgehoben und die bisherigen Publikationen der Kommission zur IVOM als obsolet bezeichnet.
\end{abstract}

Schlüsselwörter Intravitreale Injektion · Empfehlung

Recommendations of the retina committee of the Austrian Ophthalmological Society (ÖOG) for intravitreal drug administration 2017

Summary The retina committee of the Austrian Ophthalmological Society (ÖOG) has updated the last recommendations for intravitreal drug administration. The essential points that have been confirmed in studies are emphasized and the previous publications by the committee on intravitreal drug administration are obsolete.

Keywords Intravitreal injection · Recommendation

Die IVOM (intravitreale operative Medikamentenapplikation) hat sich in der Behandlung vitreoretinaler Erkrankungen als minimalinvasiver Eingriff etabliert. Schwerwiegende Komplikationen können auftreten, sind jedoch selten. Eine angepasste Vorgehensweise

Literatur beim Verfasser.

ao. Univ.-Prof. Dr. G. Kieselbach (四)

Universitätsklinik für Augenheilkunde und

Optometrie, Medizinische Universität Innsbruck,

Anichstr. 35, 6020 Innsbruck, Österreich

gerhard.kieselbach@i-med.ac.at ist aufgrund der Abhängigkeit vom injizierten Volumen oder der Substanz notwendig.

Die hier diskutierten Empfehlungen der Netzhautkommission der Österreichischen Ophthalmologischen Gesellschaft (ÖOG) sollen das Komplikationsrisiko minimieren und die IVOM als einen sicheren Eingriff in der augenärztlichen Praxis etablieren. Dieses Konsensuspapier entspricht dem Vorgehen aller Mitglieder der ÖOG Netzhautkommission.

\section{Anforderungen}

Der durchführende Arzt mit den erforderlichen Grundkenntnissen in Augenheilkunde und Optometrie hat praktische und theoretische Kenntnisse der intravitrealen Medikamentenapplikation. Die IVOM sollte in einem geeigneten Raum an einem flach gelagerten Patienten erfolgen.

Die Durchführung der IVOM in einem Operationssaal ist aufgrund der bisherigen Erfahrungen und Endophthalmitisraten in rezenten Publikationen nicht notwendig.

Die IVOM ist grundsätzlich ein ophthalmochirurgischer Eingriff.

\section{Durchführung}

Präoperativ wird eine topische Anästhesie durch mehrere Tropfen eines Lokalanästhetikums durchgeführt. Empfohlen wird die Verwendung von Einzelophthiolen („single use“).

Der häufigste Ursprung einer infektiösen postoperativen Endophthalmitis liegt in der Keimverschleppung aus der Bindehaut, Lidern und Adnexen, daher ist eine konsequente präoperative Verminderung der lokalen Bakterienbesiedlung notwendig.

Povidon-Jod besitzt bei Kontakt mit der Zellwand innerhalb von $30 \mathrm{~s}$ eine bakterizide Wirkung für die 
meisten Bakterien, zudem besteht auch Wirksamkeit bei Viren und Sporen. In einer prospektiven randomisierten doppelblinden Studie wurde der Einfluss der Konzentration auf das bakterizide Potenzial von Povidon-Jod untersucht. So konnte gezeigt werden, dass eine $5 \%$ ige Lösung eine signifikant bessere bakterizide Wirksamkeit besitzt als eine $1 \%$ ige Lösung.

Daher wird als Standard für die IVOM eine präoperative Spülung von Bindehaut, Fornices, Karunkel und Wimpern mit $2 \mathrm{ml}$ einer $5 \%$ igen Povidon-Jod-Lösung empfohlen. Eine Massage der Lider wegen möglicher Extrusion von Keimen aus den Meibom-Drüsen ist zu vermeiden.

Neben der Reduktion der patienteneigenen okulären Bakterienflora besteht ein weiterer wesentlicher Faktor zur Endophthalmitisprophylaxe in der Einhaltung von sterilen Injektionsbedingungen.

Als Standard werden

- Händedesinfektion,

- sterile Handschuhe,

- Atemmaske,

- Einsetzen eines sterilen Lidspekulums,

- Verwenden einer sterilen Kanüle

empfohlen.

Medikamente und Medizinprodukte müssen in steriler Zubereitungsform vorliegen.

Der Einstich erfolgt über die Pars plana in 3,5-4 mm Limbusabstand, um eine Verletzung von Linse oder Netzhaut zu vermeiden. Durch ein Verschieben der Bindehaut an der geplanten Injektionsstelle mit einem Tupfer wird eine Stufeninjektion durch Bindehaut und Sklera ermöglicht.

Die Injektionskanüle sollte einen Durchmesser von 27-30 Gauge und eine Länge von maximal $12 \mathrm{~mm}$ haben. In der Regel sollte der temporal untere Quadrant gewählt werden, bei wiederholten Injektionen am selben Auge sollte die Einstichstelle variiert werden. Nach Vorschieben der Kanüle 6-7 mm in Richtung auf den Sehnervenkopf zu bis zur Glaskörpermitte erfolgt die Injektion.

Im Anschluss an die IVOM soll eine Prüfung auf Lichtempfindung erfolgen. Besteht keine, wird zügig eine geeignete drucksenkende Therapie durchgeführt.

Anschließend kann ein steriler Verband appliziert werden, es gibt allerdings keine Studien, die einen positiven Effekt zeigen.

Der Nutzen einer präoperativen topischen Antibiose wird kontrovers diskutiert, und es liegen keine gesicherten Daten vor, welche einen positiven Effekt belegen können.

Das Gleiche gilt für eine postoperative topische Breitbandantibiose über mehrere Tage bei Patienten ohne erhöhtes Risiko. Entsprechend den Empfehlungen der AAO, ASRS und der rezenten Datenlage wird weder eine präoperative noch eine postoperative Antibiotikagabe bei IVOM empfohlen.

Auch bei Risikopatienten gibt es derzeit keine Hinweise, dass eine postoperative, topische Breitbandan- tibiose zusätzlich zur Antisepsis mit $10 \mathrm{ml}$ PovidonIod $5 \%$ einen Benefit erbringt. Im Gegenteil wird vermutet, dass die wiederholte Verwendung einer Breitbandantibiose die Entstehung resistenter Keime begünstigt und das Risiko erhöht.

Ein erhöhtes Endophthalmitisrisiko liegt vor, wenn Infektionen der äußeren Augenabschnitte wie ausgeprägte Blepharitis, Hordeolum, Konjunktivitis, Keratitis oder Infektionen der Tränenwege diagnostiziert werden!

Hier ist vorher ausreichend zu therapieren, bei Lidfehlstellungen oder Lidschlussdefizit sowie bei Vorliegen einer lokalen oder systemischen, endogenen oder exogenen Immunsuppression sollte die Indikation einer IVOM nur sehr vorsichtig gestellt werden.

Bei Verwendung von Kortikosteroiden sowie bei Patienten mit bekanntem Glaukom sollte eine engmaschige postoperative Kontrolle des Intraokulardruckes erfolgen. Es gibt zudem Hinweise, dass bestimmte aVEGF-Medikamente langfristig den Augeninnendruck erhöhen können.

Eine prophylaktische Parazentese wird aufgrund des erhöhten Endophthalmitisrisikos und einer schwierigeren Injektion bei weichem Bulbus nicht empfohlen.

Dem Patienten müssen im Rahmen der OP-Aufklärung v. a. die Symptome einer postoperativen Endophthalmitis wie Rötung, Schmerzen, Sehverschlechterung oder verstärkte Blendungsempfindlichkeit erläutert werden. Dabei ist besonders zu berücksichtigen, dass bei der IVOM von Triamcinolon eine Maskierung der klassischen Endophthalmitiszeichen beschrieben wurde. Die Intervalle der postoperativen Kontrollen werden dem postoperativen Befund und dem injizierten Medikament angepasst.

Diese österreichische Empfehlung zur Durchführung einer IVOM (intravitreale operative Medikamentenapplikation) spiegelt den aktuellen Wissenstand November 2017 und stellt keinen Anspruch auf Vollständigkeit.

Zur Qualitätssicherung wird empfohlen, jedwede Komplikation unter diesem Schema der Netzhautkommission der ÖOG zu melden.

Funding Open access funding provided by University of Innsbruck and Medical University of Innsbruck.

Interessenkonflikt G. Kieselbach gibt an, dass kein Interessenkonflikt besteht.

Open Access Dieser Artikel wird unter der Creative Commons Namensnennung 4.0 International Lizenz (http:// creativecommons.org/licenses/by/4.0/deed.de) veröffentlicht, welche die Nutzung, Vervielfältigung, Bearbeitung, Verbreitung und Wiedergabe in jeglichem Medium und Format erlaubt, sofern Sie den/die ursprünglichen Autor(en) und die Quelle ordnungsgemäß nennen, einen Link zur Creative Commons Lizenz beifügen und angeben, ob Änderungen vorgenommen wurden. 